\title{
Diacronie
}

Studi di Storia Contemporanea

$\mathrm{N}^{\circ} 11,3$ | 2012

La satira fa storia. Eventi, pratiche, linguaggi

\section{Dal dissenso alla rivoluzione: satira e potere nel mondo arabo contemporaneo}

\section{Barbara De Poli}

\section{(C) OpenEdition}

\section{Journals}

\section{Edizione digitale}

URL: http://journals.openedition.org/diacronie/2739

DOI: 10.4000/diacronie.2739

ISSN: 2038-0925

\section{Editore}

Association culturelle Diacronie

\section{Notizia bibliografica digitale}

Barbara De Poli, «Dal dissenso alla rivoluzione: satira e potere nel mondo arabo contemporaneo », Diacronie [Online], $\mathrm{N}^{\circ} 11,3$ | 2012, documento 9, Messo online il 29 octobre 2012, consultato il 19 avril 2019. URL : http://journals.openedition.org/diacronie/2739 ; DOI : 10.4000/diacronie.2739 


\title{
Diacronie
}

9/

\section{Dal dissenso alla rivoluzione: satira e potere nel mondo arabo contemporaneo}

\author{
Barbara De POLI*
}

Nei regimi autoritari che distinguono le realtà politiche del Vicino Oriente e Nord Africa, sovente l'opposizione trova spiragli tra le pieghe della censura attraverso la satira. Dall'oralità alla carta stampata ai tradizionali media audiovisivi ed ancora più con la diffusione di internet, la satira veicola messaggi di critica al Potere di grande immediatezza ed efficacia. Qui ci proponiamo non tanto di interpretare la satira alla luce della storia, ma attraverso un procedimento a contrario, di fornire attraverso la satira una chiave di lettura della realtà socio-politica araba contemporanea. Seguendo un approccio rasente l'antropologia politica, attraverso la satira leggeremo i percorsi del dissenso all'Autorità in particolare nell'ultimo decennio, sino alle rivolte che hanno mutato - e ancora stanno mutando - il volto di quelle regioni.

\section{Introduzione}

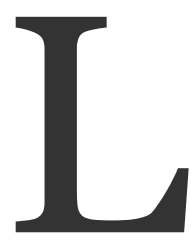

a critica ai potenti, che può essere un'arte raffinata quanto audace, trova i suoi interpreti e i suoi censori sin dall'Atene di Aristofane. Nella storia dell'Occidente, dal teatro alla letteratura colta a quella popolare, dai tradizionali strumenti audiovisivi ai nuovi media, la leva della satira ha pungolato e stimolato l'opinione pubblica mettendo a nudo mancanze, ipocrisie e contraddizioni di chi governa ${ }^{1}$, chiamando così a cambiamenti politici e sociali. Indubbiamente la satira da sola non ha mai scatenato rivoluzioni: è più il riflesso catartico di un comune sentire che il moto precursore della rivolta, anche se, come osserveremo, può diventare un'arma preziosa della mobilitazione politica. Del resto, il

${ }^{1}$ HODGART, Matthew, La satira, Milano, Il Saggiatore, 1969; LARSEN, Egon, Wit is a weapon: The political Joke in History, London, Frederick Muller Ltd., 1980. 
fatto che il potere, specie se autoritario, debolmente legittimato e deficitario sul piano politico, si preoccupi di oscurare la critica umoristica sempre con costanza e fermezza è di per sé indice del potenziale destabilizzatore di quest'ultima, capace al contempo di denunciare le tare dei governi e di sminuirli ridicolizzandoli.

Nel mondo arabo il dileggio nei confronti delle autorità era praticato sin dall'epoca classica. Si sono tramandati diversi repertori di facezie che prendevano di mira anche califfi e governanti ${ }^{2}$ o raccolte, come il Libro di Kalila e Dimna di Ibn al-Muqaffa ${ }^{3}$ (VIII secolo), in cui i rapporti di forza coi potenti venivano allegorizzati attraverso la favolistica. La fase moderna dell'umorismo politico - e della moderna censura - può invece essere fatta risalire ai tempi dello sbarco Napoleonico in Egitto, quando, stando alla testimonianza dello storico Abdel Rahmàn Jabarti (1754-1822), oltre a dover sedare rivolte armate nelle principali città, i soldati di Napoleone si trovarono costretti a perseguire ed arrestare nei mercati, nei caffè e nei hammam - pare senza grossi risultati - quanti si divertivano a deridere l'esercito francese con barzellette4.

Nei decenni a venire, satira e censura - nelle fattispecie il giornalismo satirico che andava diffondendosi insieme alla stampa - andarono di pari passo, diventando fenomeni organici del fatto coloniale, quando sia francesi che britannici si preoccupavano di sopprimere le espressioni di dissenso. Ad esempio, l'egiziano Yaqub Sanua, che nel 1877 fondò il primo giornale umoristico del mondo arabo («Naddara Zarqa» - Quello dagli occhiali azzurri), scontò con l'esilio in Francia gli attacchi che dalle sue pagine rivolgeva contro il kedivè d'Egitto e l'amministrazione britannica. La vignetta seguente è esemplificativa della carica satirica dell'epoca. Opera di uno dei primi caricaturisti egiziani, Abdel Hamìd Zaki, apparve su «The Cairo Punch», una rivista di "politica illustrata" edita tra il 1908 e il 1923, e raffigura il Console Generale britannico Eldon Gorst come un burattinaio che, secondo il noto principio dell'amministrazione coloniale indiretta, regge le fila dei diversi ministeri egiziani ${ }^{5}$

\footnotetext{
${ }^{2}$ Si rimanda ad esempio a FENOGLIO, Irène, GEORGEON, François (a cura di), Revue du Monde Musulman et de la Méditerranée : L'humour en Orient, 77-78, 1995; CATTAN, Henry (a cura di), The Gadren of Joys: An Anthology of Oriental Anecdotes, Fables and Proverbs, London, Saqi, 2000; CORRAO, Fancesca, Giufà il furbo, lo sciocco, il saggio, Milano, Mondadori, 1991; ID., Il riso, il comico e la festa al Cairo nel XIII secolo: Il teatro delle ombre di Ibn Daniya, Roma, Ipo, 1996; ROSENTHAL, Franz, Humor in early Islam, Leiden, E.J. Brill, 1956.

3 Per questo contributo abbiamo adottato una traslitterazione dall'arabo semplificata.

4 ZANELLI, Patrizia, «Egitto, mitica terra della risata», in BRANCA, Paolo, DE POLI, Barbara, ZANELLI, Patrizia, Il sorriso della Mezzaluna, Roma, Carocci, 2011, pp. 103-141; sulla satira politica in generale, KISHTAINY, Khalid, Arab Political Humour, London, Quartet Books, 1985. 5 L'immagine, estratta da http://www.cosmeo.com/viewPicture.cfm?guidImageId=AoE682AE2C9B-407F-AD20-79CA62939DA4\&\&nodeid=, è riportata in ZANELLI, Patrizia, op. cit., p. 116.
} 
Altri periodici apparsi in Egitto a cavallo tra i due secoli, caratterizzati da un'analoga vena critica umoristica, quali «Misbah al-Sharq» (La luce dell'Oriente),

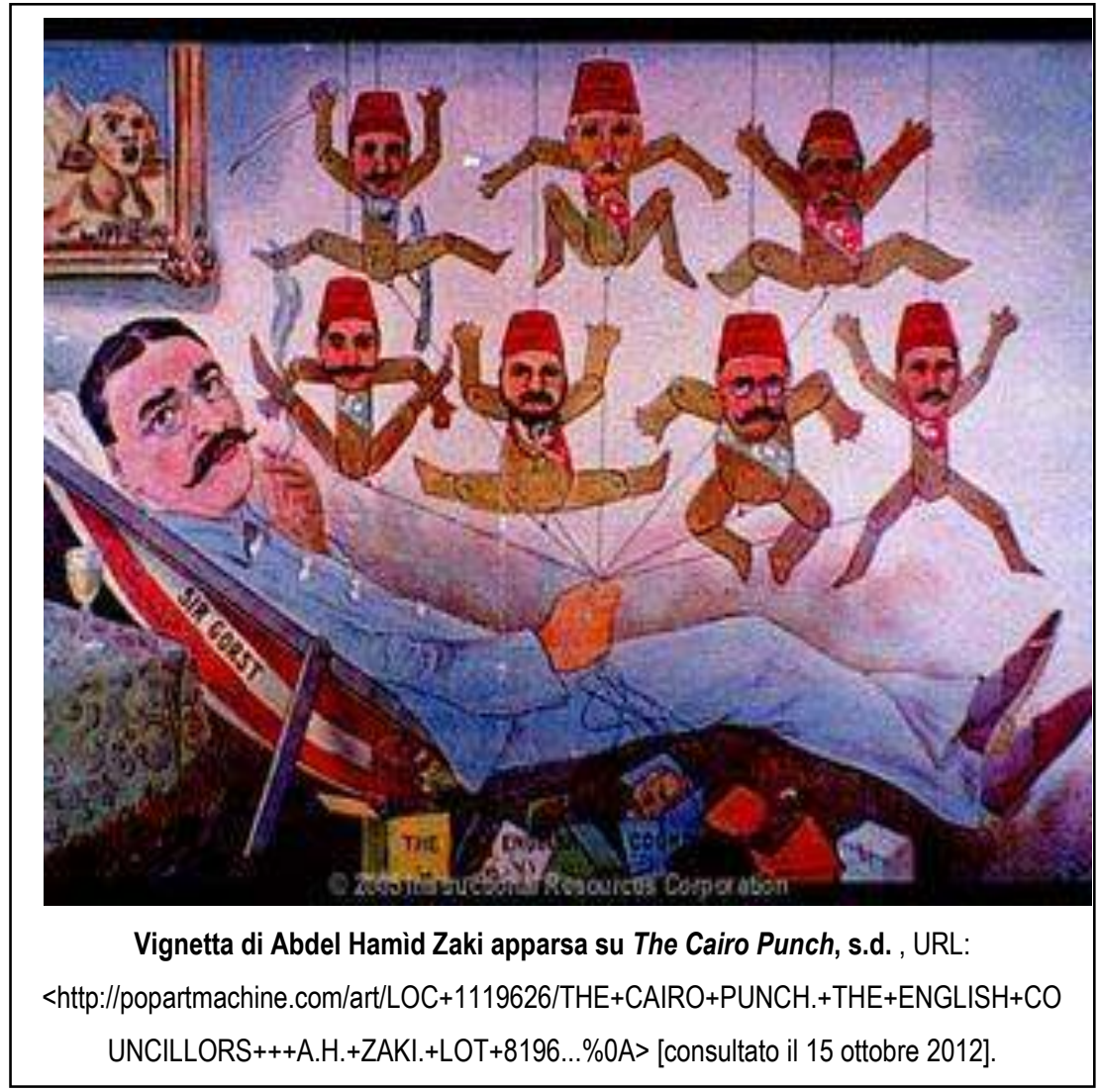

«Kayal al-zil» (Marionette dell'ombra), «Al-Sayf» (La spada), «Al-Siyasa alMusawwara» (La politica illustrata), incontrarono la censura durante la Grande guerra. $\mathrm{E}$ anche il più famoso foglio satirico egiziano del XX secolo, il progressista «Rose alYussef», fondato nel 1925 e tuttora nelle edicole, dovette affrontare in quell'epoca numerose cause legali e sospensioni ${ }^{6}$. Negli altri paesi colonizzati la stampa umoristica non conobbe una sorte migliore: in Siria, Iraq o nel Maghreb, i giornali satirici subirono censure e cessazioni. «Al-Mudhik al-Mubki» (Il tragicomico), fondato in Siria nel 1929, nei suoi 36 anni di pubblicazione, fu censurato dalle autorità francesi per complessivi dieci, riuscendo nel complesso a sopravvivere; Michael Tays, fondatore della prima testata satirica di Baghdad, fu invece assassinato nel 1925 in circostanze poco chiare, a pochi mesi dall'uscita del primo numero del suo giornale. In Tunisia non doveva andare molto meglio: nel 1932, in seguito alla fondazione di «Al-Shibàb» (La gioventù), il

${ }^{6}$ Uno dei suoi vignettisti, Abd al-Minìm Rakha, scontò un anno di prigione e, nel 1933, l'editore Muhammad al-Ta'abi, dopo i ripetuti attacchi all'amministrazione britannica, venne condannato a quattro mesi di carcere. Il settimanale sopravvisse poi alla rivoluzione dei Liberi Ufficiali e ai regimi successivi allineandosi al Potere. Dopo la caduta di Mubarak ha assunto una posizione di critica contro il Presidente decaduto, schierandosi in questo caso col comune sentire. 
poeta egiziano Bayram al-Tunsi, già espulso dal suo paese per ragioni politiche, venne arrestato ed esiliato in Francia e il periodico fu bandito; analogamente vennero censurati i giornali ad esso ispirati, che attaccavano l'occupazione straniera e chiedevano l'indipendenza7.

Il conseguimento dell'indipendenza non mutò le sorti della satira politica. Quasi ovunque nel Vicino Oriente e in Nord Africa si insediarono regimi autoritari, sovente di carattere militare, che si premurarono in primo luogo di nazionalizzare i media o perlomeno di 'addomesticarli' sopprimendo qualsiasi forma di reale opposizione, con poche eccezioni ${ }^{8}$.

Eppure, nonostante il quadro di oggettiva difficoltà espressiva, la satira contro il potere ha continuato il suo corso, anche solamente tramite l'oralità popolare, sfuggente e imprendibile strumento dell'eversione virtuale ben prima dell'invenzione di internet. Per tale ragione, nelle pagine che seguono, illustreremo il rapporto tra satira e potere prevalentemente - ma non unicamente - attraverso le barzellette, che in tal senso divengono fonte primaria della percezione popolare della politica. Non intendiamo in questa sede proporre uno studio di sociologia del folclore ${ }^{9}$, ma ci prefiggiamo di esporre alcuni temi nodali della satira contro l'autorità nei paesi arabi che vanno a caratterizzare l'autorità stessa, e di individuare alcuni specifici usi dell'umorismo satirico quale strumento di resistenza e opposizione politica.

\section{La politica araba vista attraverso la satira}

In uno dei primi giorni della sua presidenza, Sadat sta viaggiando in macchina, quando, arrivato a un incrocio, l'autista gli chiede:

- Da che parte andiamo? A destra o a sinistra?

- Hmm... Da che parte andava di solito Nasser?

- A sinistra.

\footnotetext{
$7 \mathrm{Su}$ stampa satirica e censura in epoca coloniale si rimanda in particolare a KISHTAINY, Khalid, op. cit., pp. 69-99.

8 Ad esempio il Libano, o l'Algeria degli anni '8o, prima dello scoppio della guerra civile. Cfr. HISSEIN, Amin, «Freedom as a Value in Arab Media», in Political Communication, 19, 2/2002, pp. 125-135; HAUGBOLLE, Sune, «That Joke isn't Funny Anymore: Bass Mat Watan's Nasrallah Skit and the limits of laughter in Lebanon», in Arab Media \& Society, 3/2007, URL:

$<$ http://www.arabmediasociety.com/?article $=412>$ [consultato il 17 gennaio 2011]; KHELLADI, Aïssa, «Rire quand même: l'humour politique dans l'Algérie d'aujourd'hui», in FENOGLIO, Irène, GEORGEON, François (a cura di), op. cit., pp. 225-237.

9 Per un approccio metodologico allo studio della satira come storia e sociologia del folclore si rimanda a SHEHATA, Samer, "Nasser, Sadat and Mubarak in Egyptian Political Jokes», in Folklore, 103, 1/1992, pp. 75-91, in particolare pp. 77-79.
} 
- Ah! Allora tu metti la freccia a sinistra, ma poi va' a destra!10

Poche barzellette dei tempi di Sadat hanno l'efficacia sintetica di questa: Sadat diede corso a una strategia politica e sociale antitetica a quella che aveva contraddistinto il suo predecessore, tentando tuttavia, almeno nella retorica, di attribuirsi una parvenza di continuità ${ }^{11}$. Al contempo la barzelletta allude implicitamente ai pericoli di tale pratica: mettere la freccia in una direzione e svoltare poi nell'altra può provocare seri incidenti, disorientando i veicoli che seguono e precedono, nonché i pedoni che si trovino ad attraversare in quel momento la via. Le ambiguità politiche di Sadat furono, come si sa, fatali in primo luogo per egli stesso: sebbene abbia riaperto il dialogo con gli islamisti in funzione antisocialista, il 6 ottobre del 1981 venne ucciso proprio da un estremista islamico.

Dal punto di vista della sintesi politica, la battuta su Sadat non è un'eccezione. Una carrellata anche breve su alcune note barzellette di lungo corso nei paesi arabi ${ }^{12}$ può consentire di estrarne le principali criticità dei regimi, i fattori che, in ultima analisi, hanno contribuito a produrre quella che i media occidentali hanno definito primavera araba, l'ondata di rivolte che ha portato alla caduta di Ben Alì in Tunisia, di Mubarak in Egitto, di Gheddafi in Libia e di Saleh in Yemen, mentre in Siria ha innescato un feroce conflitto interno dagli esiti al momento incerti.

Proprio in Siria è a lungo circolata la seguente barzelletta, che ben esemplifica la pervasività dei regimi che guidano/guidavano le sorti delle popolazioni arabe.

La guida turistica: - Questa è Damasco.

Il turista: - Cos'è quella?

- Quella è la biblioteca Asad

- Cos'è quello?

- Quello è il ponte Asad

- E quelli?

- Sono i giardini Asad

- ... Lo dovete amare proprio tanto il vostro Presidente! ${ }^{13}$

La battuta risale ai tempi di Hafez al-Asad, padre dell'attuale presidente siriano, e mette in luce l'identificazione della Nazione col Presidente, il quale dava il nome alle

10 Riportata in ZANELLI, Patrizia, op. cit., p. 126.

${ }_{11}$ Per una storia dell'Egitto contemporaneo, cfr. CAMPANINI, Massimo, Storia dell'Egitto contemporaneo: dalla rinascita ottocentesca a Mubarak, Roma, Edizioni Lavoro, 2005; KEPEL, Gilles, Il Profeta e il Faraone, Roma, Laterza, 2006; VATIKIOTIS, Panayiotis J., The History of Modern Egypt, from Muhammad Ali to Mubarak, London, Weindenfeld and Nicolson, 1991.

${ }_{12}$ Alcune passano indenni diversi regimi, o originate in un paese si trasferiscono in altre realtà regionali cambiando semplicemente i nomi dei protagonisti.

${ }_{13}$ Riportata in BRANCA, Paolo, DE POLI, Barbara, Ridere oggi: le barzellette, in BRANCA, Paolo, DE POLI, Barbara, ZANELLI, Patrizia, op. cit., p. 69. 
vie, ai parchi e alle principali istituzioni pubbliche, e la cui effige - così come avveniva e avviene in regimi analoghi - era ossessivamente riprodotta in ogni dove. Ovviamente la barzelletta è trasferibile tale e quale all'odierno regime del figlio Bashàr e quanto alla risposta, è data dalla guerra civile in corso.

Un altro fattore macroscopico e trasversale ai paesi arabi, che traspare dall'umorismo popolare, è la corruzione. Il fenomeno, considerato una delle principali cause delle recenti rivolte arabe ${ }^{14}$, ha stimolato la vena comica di molti. La seguente barzelletta, di matrice egiziana, è applicabile a qualsiasi realtà della regione:

\author{
Un commerciante ha tre figli: uno è intelligente, l'altro è intelligentissimo e il terzo è un \\ genio. Il padre chiede a ciascuno di loro quanto gli basterebbe avere al mondo. \\ Il figlio intelligente risponde: \\ - Io vorrei avere un milione di lire. \\ Quello intelligentissimo dice: \\ - Io vorrei avere cento milioni di lire. \\ Il genio pensa invece un po' prima di rispondere: \\ - A me basterebbe l'immunità politica ${ }^{15}$.
}

Anche le seguenti vignette, pubblicate in un settimanale satirico marocchino ${ }^{16}$, possono avere un'applicazione molto estesa:

\footnotetext{
14 Le rivolte che hanno attraversato il Vicino Oriente e il Nord Africa dalla fine del 2010 sono il prodotto di una complessità di fattori. La crisi economica legata alla globalizzazione e alle politiche liberiste che hanno depauperato soprattutto i ceti medi, si è sommata a problematiche politiche e sociali endemiche determinate dai regimi autoritari in carica da decenni: assenza di libertà di espressione, di libera competizione politica, di diritti civili essenziali, repressione poliziesca, clientelismo, corruzione. Sulle rivolte arabe cfr., ad esempio, Limes: Rivista italiana di geopolitica: Il grande Tsunami, 1/2011; Limes: Rivista italiana di geopolitica: (Contro) Rivoluzioni in corso, 3/2011; LAIPSON, Ellen (a cura di), Seismic Shift: Understanding Change in the Middles East, Stimson Center, Washington 2011; CORRAO, Francesca Maria, Le rivoluzioni arabe: La transizione mediterranea, Milano, Mondadori Università, 2011.

${ }^{15}$ BRANCA, Paolo, DE POLI, Barbara, Ridere oggi, cit., p. 70.

${ }^{16}$ Il settimanale «Demain» apparve per la prima volta nelle edicole marocchine l'11 marzo 2000 proponendosi come periodico innovativo basato su un giornalismo di inchiesta trasparente e privo di autocensure. In seguito alla prima interdizione, il giornale fu rifondato nel febbraio 2001 come «Demain magazine» e assunse una maggiore connotazione satirica, mentre nel novembre 2002 iniziò la pubblicazione del suo omologo in arabo «Dumàn», sino alla definitiva cessazione di entrambi nell'aprile 2003. La censura si accanì in particolare contro il direttore Ali Lmrabet, condannato nel maggio 2003 a quattro anni di carcere per vilipendio alla monarchia, attentato alla salvaguardia dell'integrità territoriale e al regime monarchico, dando luogo ad un affaire di risonanza internazionale. Dopo un lungo sciopero della fame Lmrabet fu rilasciato per grazia reale nel 2004. Vedi DE POLI, Barbara, Umorismo e censura nel mondo arabo: il caso 'Demain', in BRANCA, Paolo, DE POLI, Barbara, ZANELLI, Patrizia, op. cit., pp.143-176.
} 

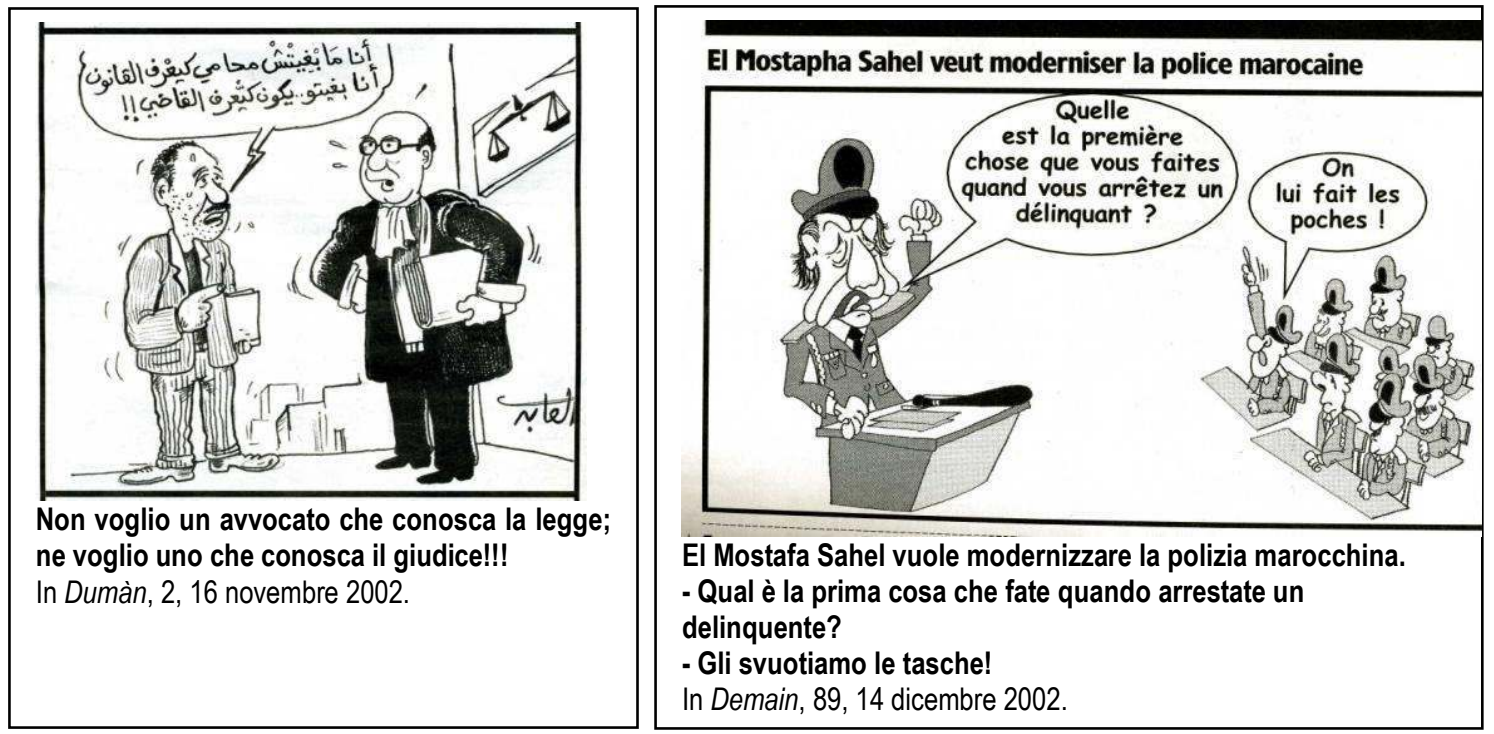

La satira contro la corruzione anticipa i dati confermati dalle statistiche: nel 2010 l'indice di percezione della corruzione dei paesi travolti dalle rivolte popolari varia dal 4.3 su $10^{17}$ della Tunisia ( $53^{\circ}$ posto su 178 ) al 3.1 dell'Egitto $\left(98^{\circ}\right)$ al 2.5 della Siria $\left(127^{\circ}\right)$ al 2.2 della Libia $\left(142^{\circ}\right)^{18}$. I modelli autoritari non si reggono su ingegnerie sociopolitiche fondate su diritti e doveri ma su favori e privilegi, sistemi clientelari di distribuzione del potere e delle risorse che coinvolgono le alte sfere come il singolo cittadino. Ricordiamo che il giovane Muhammad Bouazizi che il 17 dicembre 2010 si diede fuoco sulla piazza di Sidi Bouzid scatenando la rivolta tunisina, compì tale gesto esasperato dal taglieggiamento della polizia, che gli negava l'autorizzazione di venditore ambulante e al contempo gli consentiva di esercitare l'attività abusivamente dietro il ricorrente pagamento di un "pizzo".

L'assenza di libertà di espressione è un'altra caratteristica dei regimi arabi che l'umorismo popolare addita insieme alla severa repressione poliziesca.

Dio manda l'angelo della morte a prendere l'anima di un presidente arabo, poiché è arrivata la sua ora. L'angelo parte, ma tarda a tornare. Il Signore è irritato per il ritardo e quando finalmente l'angelo fa ritorno - solo - lo vede pesto, con un occhio nero e le ali fracassate. «Dove sei stato? Perché ci hai messo tanto? E l'anima che dovevi portarmi dov'è?». «Signore - risponde l'angelo - mi han catturato quelli dei servizi segreti. Mi hanno picchiato e continuavano a chiedermi:

\footnotetext{
${ }_{17}$ Dove 10 indica un tasso nullo di percezione della corruzione.

18 Per un confronto, la Danimarca la Nuova Zelanda e Singapore sono al primo posto con 9.3, mentre l'Italia si trova al 67 posto con 3.9. TRASPARENCY INTERNATIONAL ITALIA, CPI 201O, URL: < http://www.transparency.it/upload_doc/CPI2010_table.pdf > [consultato il 24 luglio 2012].
} 
chi sei? Cosa sei venuto a fare? Chi ti manda?». Dio, spaventato, gli chiede: «Non gliela avrai mica detto, spero?!»19

Durante una seduta parlamentare Sadat non riesce a trovare la penna che usa sempre per firmare. Chiede perciò al ministro degli interni di cercarla. Quando però poi va a casa, lui stesso la trova in mezzo alle sue carte. Allora telefona al ministro e gli dice:

- È tutto a posto, ho trovato la penna.

- Ma, signore, che ne facciamo adesso dei dieci che hanno confessato di averla rubata?!20

Due amici si incontrano per strada, e uno di loro dice all'altro:

- Hai saputo di tizio? Si è fatto togliere un dente attraverso il naso!'

- Perché dal naso?'

- E chi mai può aprire bocca da queste parti?21

Nel Vicino Oriente e in Nord Africa la stampa conosce una generale irreggimentazione e qualsiasi voce del dissenso politico viene tacitata con un controllo e una repressione capillari. Il regime poliziesco era particolarmente noto per la sua ferocia in Tunisia e in Siria, ma anche in Egitto, malgrado le aperture di Mubarak, gli abusi da parte delle forze dell'ordine erano moneta corrente - anche se con effetti talvolta opposti a quelli desiderati. Il brutale assassinio da parte della polizia egiziana del ventottenne Khalid Said, all'uscita da un cybercafè nel giugno 2010 probabilmente perché in possesso di materiale video che implicava le forze dell'ordine in traffici di droga -, ispirò la pagina Facebook di Wail Ghoneim intitolata a suo nome: «Siamo tutti Khalid Said». Il sito, che denunciava la corruzione del regime, fu uno dei più importanti catalizzatori virtuali del dissenso poi sfociato nella rivolta di Piazza Tahrìr.

Ovviamente le barzellette non trascurano le questioni economiche. Le difficoltà che oggi attraversano soprattutto i paesi arabi non produttori di petrolio hanno radici lontane, ma sono state accentuate e accelerate dalla globalizzazione e dalle politiche liberali dell'ultimo decennio, che impoverendo ulteriormente le masse hanno prodotto la rottura di qualsiasi patto sociale preparando il terreno alle rivolte. È un dato

\footnotetext{
19 BRANCA, Paolo, DE POLI, Barbara, Ridere oggi, cit., p. 66.

20 Ibidem, p. 69.

${ }^{21}$ In particolare questa barzelletta conosce innumerevoli varianti (la più frequente sostituisce l'intervento alle tonsille all'estrazione di un dente). Cfr. Ibidem, p. 73 e ZANELLI, Patrizia, op. cit., p. 124.
} 
condiviso dagli analisti che tra i fattori a monte degli eventi del 2011, la crisi economica sia stata determinante almeno in Tunisia e in Egitto, così come ha certamente posto le basi per il conflitto siriano ${ }^{22}$.

La prima barzelletta di seguito riprodotta risale ai tempi di Nasser:

Il ministro degli approvvigionamenti sta facendo un giro d'ispezione dei forni per controllare la qualità del pane. Quindi, chiede a una casalinga:

- Che ne pensa di questo pane, Signora?

- Secondo me, non è buono per niente.

- Ah! Ma lo sa che ai nostri antichi avi piaceva molto questo pane?

- E ci credo, almeno a quei tempi sarà stato fresco! 23

La vignetta che segue è invece marocchina e risale ai primi anni di governo di Muhammad VI, inizialmente detto anche "Re dei poveri", in ragione delle tante aspettative sulle sue politiche sociali ${ }^{24}$.

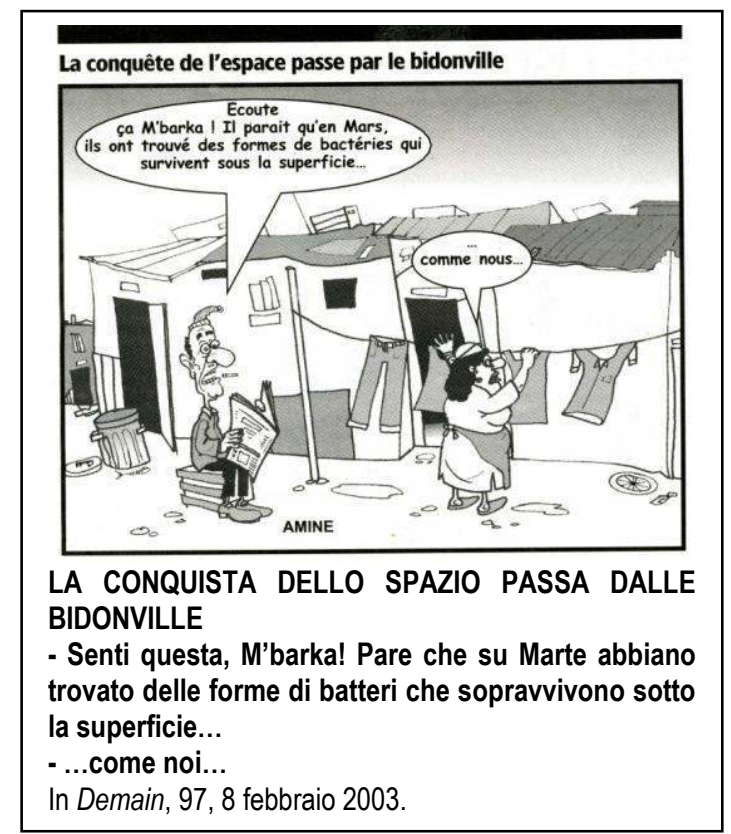

${ }^{22}$ Sul rapporto tra rivolte e fattori economici vedi ABDIH, Yasser, «Arab Spring: Closing the Jobs Gap. High youth unemloyment contributes to widespread unrest in the Middle East», in Finance \& Development, International Monetary Fund, June 2011, pp. 36-39, URL: < http://www.imf.org/external/pubs/ft/fandd/2011/o6/pdf/abdih.pdf > [consultato il 5 agosto 2012] e, soprattutto, TRENTIN, Massimiliano, «Le rivolte arabe tra storia, economia e politica», Europa e Mediterraneo, Pisa, ETS, in corso di stampa.

23 ZANELLI, Patrizia, op.cit., p. 125.

24 Malgrado un movimento di contestazione abbia preso piede anche in Marocco, il paese subisce in modo marginale l'effetto domino della primavera araba, in ragione della forte legittimazione popolare della monarchia, di un progresso dei diritti civili e della partecipazione democratica nell'ultimo decennio, del progressivo, seppur modesto, miglioramento delle condizioni economico e sociali delle masse. Vedi LÓPEZ GARCÍA, Bernabé, Le Maroc et le Printemps Arabe dans un monde en plein changement, Barcellona, IEMed, 2012, pp. 40-42. 
Un altro dato che emerge con chiarezza dall'umorismo popolare arabo è la scarsa stima nei confronti di chi governa. Le barzellette ci spiegano che i regimi si reggono sull'uso della violenza e della prevaricazione, senza godere di alcuna considerazione da parte dei governati.

Un arabo viene processato per aver esclamato in pubblico: "Governo di merda!". Per difendersi dichiara: "Ma io non mi riferivo al nostro!" Il giudice tuttavia lo condanna lo stesso: "Col governo che abbiamo, non poteva intendere altro!"25.

Il Presidente Mubarak riceve in regalo un puzzle gigante. Si chiude nel suo studio intimando ai suoi collaboratori di non disturbarlo per nessun motivo finché non abbia terminato il puzzle. Passano un mese, due, tre, un anno, finalmente il Presidente esce dallo studio, con la barba lunga, sporco, stremato e convoca immediatamente il Consiglio dei ministri: "Avete visto? Ho impiegato solamente un anno a risolverlo, mentre sulla scatola c'è scritto ‘dai tre agli otto anni' ${ }^{26}$.

Alla luce dei contenuti della satira quotidianamente praticata contro i loro governi, la rabbia, l'esasperazione e la determinazione dimostrate dalle masse scese in piazza dal dicembre 2010 stupiscono meno. E un fattore politicamente secondario ma sociologicamente e antropologicamente significativo riguarda proprio l'uso della satira durante le rivolte di piazza e nei processi di transizione politica tutt'oggi in corso: soprattutto in Egitto e Tunisia, l'inattesa e incontenibile contestazione di massa contro i regimi si è sprigionata anche attraverso una dirompente carica umoristica collettiva.

\section{Satira e rivoluzione}

«Il comico è strumento di conservazione, diverge le energie contestatarie, acqueta le irritazioni, i grandi rivoluzionari non sapevano ridere e guai se si fossero fermati a ridere» ${ }^{27}$. Se Umberto Eco avesse assistito alle rivolte di Tunisi e del Cairo del 2011 forse si sarebbe espresso diversamente. In realtà già Srdja Popovic, uno dei fondatori del movimento pacifista serbo Otpor che contribuì alla caduta del regime di Milosevich nel 2000, aveva coniato l'espressione laughtivism, a indicare l'impiego dell'umorismo

25 BRANCA, Paolo, DE POLI, Barbara, Ridere oggi, cit., p. 71.

${ }^{26}$ Ibidem, p. 66.

${ }_{27}$ ECO, Umberto, «Il nemico dei filosofi», in L'Espresso, 13 agosto 1967, p. 18. 
nella mobilitazione politica non-violenta, cosa di cui Otpor fece un uso 'scientifico' ed incisivo.

Se è indubbio che, tra le forme umoristiche, la satira è uno strumento capace di pungolare gli spiriti e sollevare riflessioni sul potere, è ancor più vero che la caduta di un regime repressivo o la prospettiva della sua caduta possono improvvisamente dare la stura ad una pressione umoristica da tempo repressa. Le rivolte che nel corso del 2011 hanno portato in Nord Africa alla caduta di regimi decennali, con esiti ancora incerti, hanno anche fatto erompere l'ironia collettiva. Ciò non significa che prima di quel momento, come illustrato nel precedente paragrafo, non si dessero forme di resistenza umoristiche e principalmente satiriche ${ }^{28}$, ma durante le manifestazioni gli attacchi derisori contro i regimi divennero un fenomeno macroscopico, parte integrante della protesta, e gli scherni gridati sulla pubblica piazza ripresi dai network internazionali, si proiettavano sui monitor del mondo intero assumendo dimensione planetaria. L'esplosione satirica è stata forse più evidente in Egitto, paese a speciale vocazione umorista per indole e tradizione ${ }^{29}$, ma si è registrata analogamente in Tunisia.

Nel paese maghrebino, l'avvio della rivolta fu battezzato dall'umorismo. Il 18 dicembre 2010, giorno successivo a quello del tragico gesto di Muhammad Bouzizi e considerato la data d'inizio della rivoluzione, su Facebook rimbalzava già la seguente battuta:

La prima misura presa da Ben 'Alì: vietata la vendita di fiammiferi a Sidi Bouzid3o.

Da quel momento le reazioni umoristiche di piglio satirico o anche sarcastico accompagneranno quasi sincronicamente ogni atto del regime. Quando ad esempio, il

${ }_{28}$ Per quanto riguarda in particolare l'Egitto, cfr. HAMÙDA, Àdel, Al-Nukta al-siyàsiyya: kayfa yaskhar al-misriyyùna min hukkàmihim [Le barzellette politiche: come gli egiziani prendono in giro i loro governanti], Cairo, Fursàn li'l-Nashr, 1990; KAMIL, Àdel, al-Kàrikàtìr fi Misr (La caricatura in Egitto], Cairo, Maktabat al-Usra, al-Hay'a al-Misriyya al-Amma li'l-Kitàb, 2009; EL AMRANI, Issandr, «Three Decades of a Joke That Just Won't Die», Foreign Policy, January/February 2011, URL:

<http://www.foreignpolicy.com/articles/2011/o1/o2/three_decades_of_a_joke_that_just_wo nt_die > [consultato il 4 luglio 2012]; SHEHATA, Samer, op. cit.

${ }_{29}$ ZANELLI, Patrizia, op. cit. Adel Iskandar studioso di media di origine cairota spiega in un'intervista: «Not only can I not imagine a revolution in Egypt without jokes, I cannot imagine anything in Egypt without jokes»: SUSSMAN, Anna Louie, «Laugh, O Revolution: Humor in the Egyptian Uprising», in The Atlantic, 23 February 2011, URL:

$<$ http://www.theatlantic.com/international/archive/2011/o2/laugh-o-revolution-humor-inthe-egyptian-uprising/71530/ > [consultato il 10 luglio 2012].

30 ZIZIRIDER69, «Révolution Tunisienne et politique (Humour)», in Les élucubrations de Zizirider, URL: < http://zizirider.blogspot.it/p/revolution-tunisienne-humour.html > [consultato il 12 luglio 2012]. 
10 gennaio Ben Alì annunciò la creazione di trecentomila di posti di lavoro e destituì il ministro degli interni, il web reagì subito:

Primo posto di lavoro creato: ministro dell'interno; ne mancano ancora 299.99931.

oppure:

Attenzione, i francesi cominceranno ed emigrare clandestinamente in Tunisia per cercare lavoro ${ }^{32}$.

Di lì a pochi giorni, il contagio rivoluzionario si trasferiva in Piazza Tahrir, dove le accuse al regime assumevano le più diverse forme umoristiche, dall'ironia alla satira strisciante. Gli attacchi contro Mubarak cominciarono a colorare i muri sotto forma di graffiti, a rimbalzare nei social network con vignette o barzellette e a trasformarsi in provocazioni sugli striscioni e i cartelli dei manifestanti33.

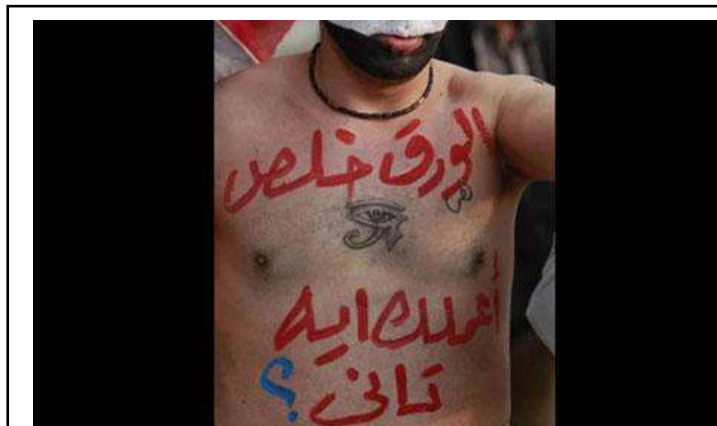

Manifestazioni in Egitto contro Mubarak. Sul petto del manifestante: «Ė finita la carta. Che altro potevo fare?».

In Ahram Online, URL:

$<$ http://english.ahram.org.eg/NewsContent/1/64/5907/Egyp t/Politics-/Egyptian-sense-of-humour-prevails-inrevolution.aspx>.

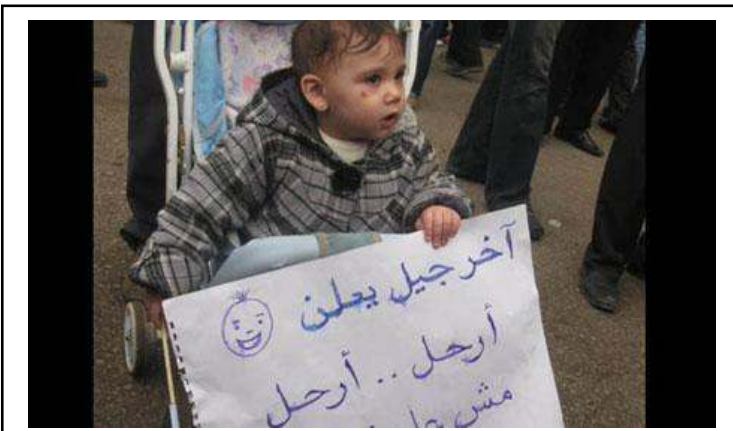

Manifestazioni in Egitto contro Mubarak. Sul cartello: "L'ultima generazione dice: vattene... vattene...». In Ahram Online, URL:

$<$ <ttp://english.ahram.org.eg/NewsContent/1/64/5907/Egypt/

Politics-/Egyptian-sense-of-humour-prevails-in-

revolution.aspx>.

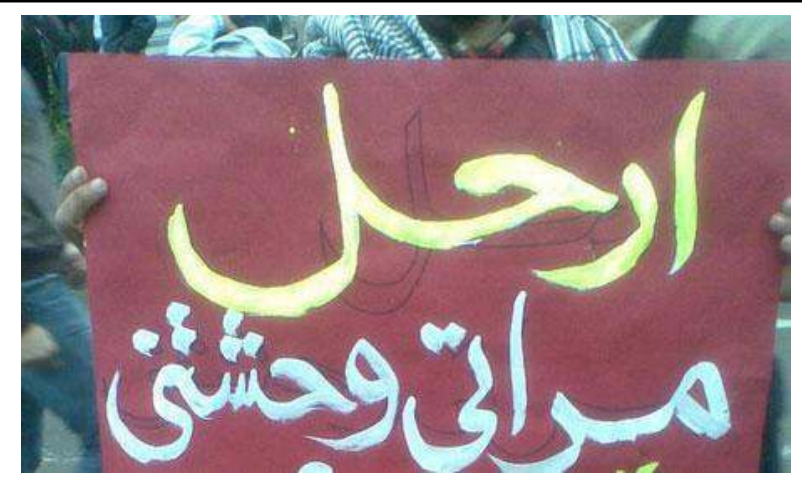

Manifestazioni in Egitto contro Mubarak. Sul cartello: "Vattene. Mi manca mia moglie». Un'altra tipologia di cartello, frequentemente ricorrente nelle proteste recava scritto: «Vattene. Mi fa male il braccio [a tenere il cartello]».

In Ahram Online, URL:

$<$ http://english.ahram.org.eg/NewsContent/1/64/590

7/Egypt/Politics-/Egyptian-sense-of-humour-

prevails-in-revolution.asp $>$.

${ }^{31}$ BEN ABDELADHIM, Maha, «L'humour au temps de la révolution», in France 24, 23 janvier 2011, URL: < http://www.france24.com/fr/20110122-tunisie-ben-ali-humour-facebookrh\%C3\%A9torique-jeunes-resistance $>$ [consultato il 2 luglio 2012].

32 Ibidem.

33 Un resoconto dei giorni della protesta costruito attraverso la satira è fornito da LAURITO, Alessandra, Satira e rivoluzione: uno studio socio-culturale sull'uso dell'umorismo nella rivoluzione egiziana del 2011, Tesi di laurea in storia dei Paesi arabi, Università Ca' Foscari Venezia, a.a. 2010-2011. 
Anche i reciproci richiami scandivano le nuove battute:

Il Presidente Ben Alì, oramai in rifugio a Geddah, dice a Mubarak: "Quando vieni ricordati di portare il joystick della playstation 34 .

Ovviamente in questo quadro i nuovi media, internet e in primo luogo i social network hanno svolto un ruolo cruciale. Com'è noto, negli anni precedenti la rivolta, gli strumenti informatici si sono rivelati un importante dispositivo di coagulo del dissenso politico in ragione dell'immediatezza della comunicazione e della maggiore difficoltà di intercettazione degli attori da parte delle forze di sicurezza35; attraverso i social network inoltre si sono lanciate e in larga misura coordinate le proteste di massa. Per gli stessi motivi i social network sono diventati il luogo di massima circolazione della satira contro il potere. La battuta che segue è diventata in tal senso iconica della rivolta egiziana:

Dopo la sua morte Mubarak incontra Sadat e Nasser. Gli chiedono come sia morto, veleno o pallottola. Mubarak irato risponde: Facebook!

Si moltiplicano così i siti che fanno da cassa di risonanza della satira antiregime ${ }^{36}$. In Egitto citiamo ad esempio pagine di informazione come «al-Shurùk», «al-Masri alYawm» e «Mashy»; di giornalismo satirico come «al-Koshary Today»; o specializzate in vignette come «Latuff2»37. In Tunisia segnaliamo giornali on-line come

\footnotetext{
34 MAHER, Hatem, «Egyptian sense of humour prevails in revolution», Ahramonline, 20 February 2011, URL: < http://english.ahram.org.eg/NewsContent/1/64/5907/Egypt/Politics/Egyptian-sense-of-humour-prevails-in-revolution.aspx > [consultato il 20 giugno 2012].

35 ALTERMAN, John B., New Media, New Politics? From Satellite Television to the Internet in the Arab World, Washington, Washington Institute for Near East Policy, 1999; FANDY, Mamoun, «Information Technology, Trust, and Social Change in the Arab World», in The Middle East Journal, LIV, 3/2000, pp. 378-394; HAFEZ, Kai, Mass Media, Politics, and Society in the Middle East, New York, Hampton Press, 2001; PASSY, Florance, GIUGNI, Marco, «Social Networks and Individual Perceptions: Explaining Differential Participation in Social Movements», in Sociological Forum, 16, 1/2001, pp. 123-153; SIBILIO, Simone, «La rivoluzione dei (nuovi) media arabi», in CORRAO, Francesca (a cura di), op. cit., pp. 81-109.

${ }^{36} \mathrm{Si}$ osservi che la rete, come evidenzieranno anche gli esempi nelle pagine successive, palesa un orientamento in larga prevalenza laico. Nondimeno si ricorda che i movimenti radicali, anche estremisti jihadisti, furono precursori nell'utilizzo di internet come strumento di comunicazione, propaganda e proselitismo. Indubbiamente i siti islamici non spiccano per verve comica. Cfr. THOMAS, Dominique, Le rôle d'internet dans le diffusion de la doctrine salafiste, in ROUGIER, Bernard (a cura di), Qu'est-ce que le salafisme ?, Paris, Puf, 2008, pp. 87-102; TROMBETTA, Lorenzo, "Il jihad elettronico», in Limes : Rivista italiana di Geopolitica : Progetto Jihād, 1/2004, pp. 43-51; EL-NAWAWY, Mohammed, KHAMIS, Sahar, Islam Dot Com: Contemporary Islamic Discourses in Cyberspace, London, Palgrave Macmillan, 2009; BUNT, Gary R., Islam in the Digital Age, London, Pluto Press, 2003.

37 Rispettivi URL: < http://www.shorouknews.com/ > ; < www.almasryalyoum.com >; <http://www6.mashy.com>; < http://elkoshary.com > ; < http://latuff2.deviantart.com > [consultati il 15 settembre 2012].
} 
«Businessnews», blog come «DebatTunisie» di ' $Z$ ' e pagine facebook, come «Ennahdafacts» 38 .

L'esplosione umoristica si è manifestata con tanta evidenza che se prima delle rivolte gli studi sull'umorismo e in particolare la satira nel mondo arabo erano alquanto infrequenti, dopo le rivolte di Tunisi e del Cairo la tendenza si inverte. Studiosi, giornalisti e blogger si sono interessati alla raccolta di barzellette e varie altre espressioni umoristiche incentrate sulle proteste, e allo studio dell'umorismo al tempo delle rivolte si sono dedicate tesi di laurea, nonché panel di convegni39.

L'umorismo politico che esplode durante le manifestazioni di piazza apportando un contributo incisivo alla lotta contro i regimi va ricondotto a più fattori.

In primo luogo la satira che denigra e disprezza il regime davanti al pubblico internazionale ne consolida la piena delegittimazione e debolezza nel confronto coi manifestanti ${ }^{4}$. In secondo luogo, in una situazione di grave tensione collettiva e di autentico rischio (sia al Cairo che a Tunisi non era prevedibile che l'esercito si schierasse con i manifestanti) ogni forma di umorismo può divenire una forma di mutua rassicurazione con funzione esorcizzante nonché tranquillizzante ${ }^{41}$; una blogger tunisina anonima conferma che: «cette dérision immédiate offre le recul et la distance nécessaires par rapport à la situation que vivent actuellement les Tunisiens. C'est l'un des moyens les plus efficaces de garder la tête froide» ${ }^{42}$. Infine, come sostiene lo stesso Popovic, per quanto riguarda i movimenti di opposizione pacifica che intendano mobilitare le masse, l'umorismo che espone l'Autorità al ridicolo è un'ottima arma per spezzare la paura dei cittadini assuefatti a regimi che proprio sulla paura si fondano 43 .

${ }^{38}$ Rispettivi URL: < http://www.businessnews.com > ; < http://www.debatunisie.com/ > ; $<$ https://www.facebook.com/ennahdhafacts $>$ [consultati il 15 settembre 2012].

39 Tra le tesi di laurea, solo in Italia, citiamo ad esempio, LAURITO, Alessandra, op. cit.; DE MARTINO, Marta, La rivoluzione egiziana attraverso le vignette di Muhammad Abdelatif, Tesi di traduzione arabo-italiano, Roma, Università LUSPIO, a.a. 2011-12; tra i convegni seminari che hanno dedicato sezioni all'umorismo, ricordiamo Translating Revolution tenuto all'Università Americana del Cairo il 21 maggio 2011 e il convegno organizzato a Beirut dall'Orient Institut, Inverted Worlds, tenutosi nell'ottobre 2012.

${ }^{40}$ Scrive Sigmund Freud in Il motto di spirito e la sua relazione con l'inconscio, nel terzo capitolo, Gli scopi dei motti di spirito: «Minimizzando quest'ultimo [il nemico], considerandolo inferiore, rendendolo spregevole o ridicolo, noi raggiungiamo per vie traverse lo scopo di sopraffarlo, della qual cosa la terza persona che non ha fatto nessuno sforzo, diviene testimone con le sue risa». FREUD, Sigmund, Il motto di spirito e la sua relazione con l'inconscio, Roma, Newton Compton, 1976, p. 110.

${ }^{41}$ Gli studi psicologici hanno dimostrato che la 'somministrazione' di umorismo è in grado di desensibilizzare dalla paura gli individui. VENTIS, W. Larry, HIGBEE, Garret, MURDOCK, Susan A., «Using humor in systematic desensitization to reduce fear», in Journal of general psychology, 128/2, April 2001, pp. 141-153.

42 BEN ABDELADHIM, Maha, op. cit.

43 HUMORCODE, Srđa Popović on the power of "laughtivism", 01 maggio 2012,

URL: < http://www.youtube.com/watch?v=4rdxk3R4NxA > [consultato il 28 luglio 2012] 
Il legame tra alcuni movimenti di opposizione egiziani e il movimento serbo Otpor è noto. Soprattutto gli attivisti del gruppo 6 aprile (Haraka 6 Abril) 44 hanno fatto appello al Center for Applied NonViolent Action and Strategies (CANVAS) 45 per informarsi sulle pratiche di resistenza e di mobilitazione di piazza pacifiche, e se qualche consiglio è stato dato anche sull'uso dell'umorismo, cadeva certamente sul bagnato, vista la lunga tradizione egiziana in tal senso. L'uso consapevole dell'umorismo da parte dei movimenti non violenti sembra confermato da più voci. "It's very effective because it breaks the fear barrier»46, assicura Mahmoud Salem, blogger chiamato Sandmonkey, intervistato sull'umorismo ai tempi della rivolta; mentre il giornalista e blogger Issandr el-Amrani conferma che «The organizers used humor as part of their communications strategy, to motivate people and bring out the crowds»47, convinto che almeno in parte l'umorismo fosse pianificato: «There was a lot of spontaneous humor - it is the Egyptian character - but there also was a desire to show that the demonstrators weren't just angry young men, that they weren't just seen as Islamists»48.

L'umorismo, dunque, e in particolare la satira usati come arma di azione nonviolenta, diventano anche il riflesso del successo di tale strategia. Una volta caduti i regimi e assunta piena consapevolezza della forza dell'opinione pubblica, la voglia di superare i modelli autoritari per dare corso a sistemi democratici e liberali si è immediatamente tradotta nell'accesso alla libera opinione anche satirica. Seppure il campo mediatico ufficiale non sia ancora totalmente aperto e molti preferiscano ancora postare le proprie vignette sui social-network ${ }^{49}$, la satira sui media è proliferata come mai in precedenza.

44Il movimento nasce nella primavera del 2008 in sostegno alle lotte operaie del distretto industriale di Mahalla el-Kubra e ha come principali piattaforme organizzative e di dibattito i social network. I rapporti col movimento serbo Otpor (del quale hanno ripreso il simbolo col pugno chiuso) sono precoci e datano tre anni prima l'avvio delle rivolte. Vedi la pagina del movimento, URL: < http://6aprilmove.blogspot.it/ > [consultato il 6 settembre 2011]; e sull'azione del gruppo nel corso della rivolta: ALJAZEERAENGLISH, People \& Power - Egypt: Seeds of change, 9 febbraio 2011, URL:

< http://www.youtube.com/watch?v=QrNzodZgqN8video > [consultato il 6 settembre 2011]. 45 Fondata nel 2004 da Srja Popović e Slobodan Djinović, fondatori del movimento serbo Otpor, l'ONG si occupa di istruire sui metodi di lotta non-violenta per promuovere i diritti umani e la democrazia. Vedi il sito dell'organizzazione: < http://www.canvasopedia.org/legacy/> [consultato il 18 ottobre 2012].

${ }^{46}$ SUSSMAN, Anna Louie, op. cit.

47 SLACKMAN, Michael, «When a Punch Line Is No Longer a Lifeline for Egyptians», in The New York Times, 4 April 2011, URL:

< http://www.nytimes.com/2011/o4/o6/world/middleeast/o6cairo.html?pagewanted=all > [consultato il 18 luglio 2012].

48 Ibidem.

49 Anche dopo la caduta di Mubarak, un vignettista indipendente come Islàm Garwìsh, (che quando il vecchio regime era ancora in carica incollava le sue vignette antigovernative su mura e palazzi), risulta scomodo e non trova spazio nei media tradizionali, che comunque sono 
Oggetto delle critiche sono divenuti gli apparati residuali ma politicamente radicati dei regimi decaduti, segnatamente i militari in Egitto, oppure, specialmente in Tunisia, gli islamisti usciti vittoriosi dalle prime libere elezioni.

In Egitto il Supremo Consiglio delle Forze Armate (SCAF) che ha gestito la fase di transizione dopo la caduta di Mubarak tentando di garantirsi una continuità politica, è diventato particolare oggetto della satira che continua a evidenziarne l'inadeguatezza.

Il graffito disegnato su un edificio vicino a Piazza Tahrirso raffigura la longa manus dell'esercito sulle elezioni presidenziali e non può non richiamare a mente la vignetta di Hamìd Zaki riferita ad Eldon Gorst, riportata sopra.

«Al-Koshary Today», forse il più noto dei fogli satirici on-line ${ }^{51}$, così commenta i primi provvedimenti politici del governo militare di transizione:

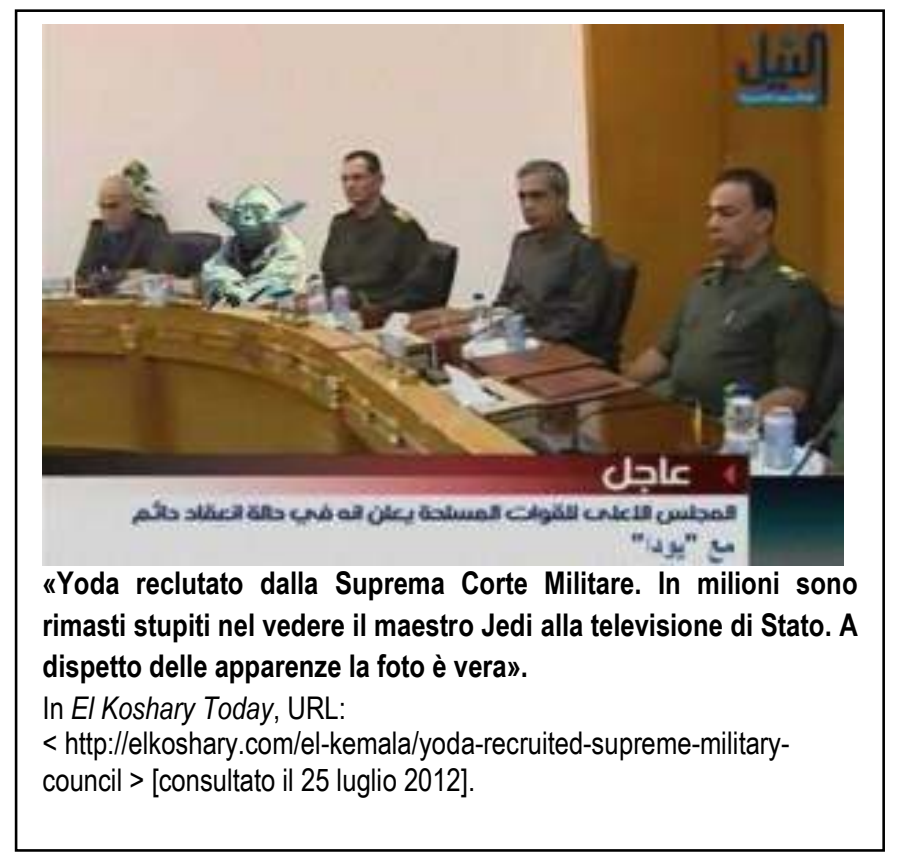

generalmente schierati e non accettano un umorismo indipendente. Preferisce raggiungere il suo vastissimo pubblico sui social network Intervista a Garwìsh in WOODSOME, Kate, ELSHINNAWI, Mohamed, «In Uncertain Times, Egyptian Comedy Thrives», in Voices of America, 9 July 2012, URL:

< http://www.voanews.com/content/egypt_political_humor/1381497.html > [consultato il 2 giugno 2012].

${ }^{0}$ L'immagine del graffito di piazza Tahrir è presente in Voice of America, URL:

<http://www.voanews.com/content/egypt_political_humor/1381497.html>.

${ }^{5}$ Fondato nel 2009, il quotidiano on-line «El-Koshary Today» (il koshary è il piatto nazionale egiziano a base di pasta riso, cipolle e legumi), si ispira ai siti di informazione governativa per fare satira proponendo notizie fasulle allo scopo di denunciare le reali storture della politica e della società. I tre che lo animano hanno sempre preferito mantenere l'anonimato usando gli pseudonimi di Makarona (maccheroni), Ward Ziyada (cipolla extra) e Subar Lox (maxi porzione di Koshary). AMER, Pakinam, «El Koshary Today: Egypt's on line 'fake news' paper», Egypt Independent, 18 november 2009, URL: < http://www.almasryalyoum.com/en/node/2723 > [consultato 25 luglio 2012]. 


\begin{abstract}
Written by Makarona
Tue, 07/05/2011 - 18:54

State TV broadcast live footage yesterday of the first meeting between the Supreme Council of the Armed Forces (SCAF) and newly appointed "Wisdom Advisor" Yoda. It remains unclear why Yoda has decided to join the dark side of the force at this time. Questions are also being raised about how the green sage - long presumed to have died in a galaxy far far away - made his way to Cairo in the 21st centry. Currently, all that is known of the meeting is that when SCAF members informed Yoda that they were trying to stop citizens from protesting, the Jedi master solemnly replied, «Do. Or Do not. There is no try». At this point the SCAF reportedly erupted into awed applause ${ }^{52}$.
\end{abstract}

La satira accusa costantemente l'azione del governo militare, allo scopo di tenere allerta l'opinione pubblica; come spiega Samer Shehata: «It is harsh, biting and particularly effective at exposing some of the lies of the SCAF and their allies presenting a more critical perspective on current political events» 53 .

Sul fronte tunisino, analogamente, sembra quasi che siano le battute a dettare la cronologia degli eventi post-rivoluzionari. Quelle che seguono sono di commento alla caduta di Ben Alì e del suo regime familistico.

Vendesi, causa trasferimento, banche, scuole, compagnie aeree e di navigazione, operatori di telefonia fissa e mobile, 4 canali radiofonici, castelli, lotti di terreno, una tigre, un salone di parrucchiere, Hammer H2, casa Volkswagen e un aereo imballato. Contattare Trabelsi 0711 1987. Prezzo trattabile54.

Alì Baba è partito, ma non i 40 ladroni! 55

$5^{2}$ In El Koshary Today, URL: < http://elkoshary.com/el-kemala/yoda-recruited-suprememilitary-council $>$ [consultato il 25 luglio 2012].

53 WOODSOME, Kate, ELSHINNAWI, Mohamed, op. cit.

54 Citata da Maha Ben Abdeladhim (op. cit.), la barzelletta fa chiaro riferimento al sistema di interessi famigliari che reggeva la presidenza, in particolare dalla moglie di Ben 'Alì, Laila Trabelsi, che notoriamente era parrucchiera, e i suoi fratelli che si sono spartiti i settori produttivi del paese. Sulla storia recente della Tunisia cfr. CAMAU, Michel, GEISSER, Vincent, Le Syndrome Autoritaire: politique en Tunisie de Bourguiba à Ben Ali, Paris, Presses de Sciences Po, 2003; HIBOU, Beatrice, La force de l'obeissance: économie politique de la répression en Tunisie, Paris, La Découverte, 2006; PERKINS, Kenneth J., A History of Modern Tunisia, Cambridge, Cambridge University Press, 2004. Sul sistema clientelare di Ben Alì e della moglie Leila Trabelsi cfr. BEAU, Nicolas, GRACIET, Catherine, La régente de Carthage, Paris, La Découverte, 2009; BEAU, Nicolas, TUQUOI, Jean Pierre, Notre ami Ben Ali, Paris, La Découverte, 2002.

55 Questa e le due barzellette successive sono citate in più siti:

<http://www.hanafi-art.com/revolutiontunisienne4.html> ;

<http://www.google.com/hostednews/afp/article/ALeqM5hhzmoH1CrKFwjEYUQoMzfh-EaKA?docId=CNG.54fe291edb146dd9b8a4f8848b443680.91> ;

<http://www.rue89.com/2011/01/21/la-tunisie-decouvre-les-blagues-politiques-186708>

[consultati il 20 luglio 2012] 
Offerta d'impiego n. 140111 : Cercasi nuovo presidente per la Tunisia: si accettano apprendisti. Tipo di contratto: a termine. Qualità richieste: orfano, figlio unico, sterile soprattutto calvo di modo che non frequenti parrucchieri.

Prima avevamo un presidente ogni ventitré anni, adesso ne cambiamo uno al giorno56.

In Tunisia, con l'andare dei giorni, date le oggettive difficoltà di costruire un sistema democratico i toni dell'umorismo cambiano. In particolare, il rientro in Tunisia di Rachid al-Ghannouchi, ideologo del partito di orientamento islamico Ennahda, e l'avvio della campagna per l'elezione dell'Assemblea Costituente daranno luogo ad uno scontro non solo ideologico tra laici e radicali57, riflesso dalla satira on-line che esterna un piglio spiccatamente anti-islamista. A ottobre, la vittoria di Ennahda (col 37\% dei suffragi) esacerberà i toni umoristici, sovente sarcastici, contro la cultura radicale propugnata dagli islamisti e le derive sempre più evidenti del governo da essi dominato.

Facebook, 2 aprile 2011: la rivoluzione tunisina partorirà un topolino che si trasformerà presto in un tirannosauro peggiore di Ben Alì 58 .

Twitter, 8 agosto 2011: La poligamia in Tunisia, ovvero: 4 mogli di Presidente, 4 Leila [Trabelsi] in potenza; accidenti è un disastro, specie se hanno 13 fratelli ciascuna.

Twitter, 10 agosto 2011: Il 13 agosto, festa della donna organizzata da Ennahda: programma di dimostrazione di lapidazione e violenza coniugale perché 'voi lo valete'.

Il dissenso contro il partito islamista dilaga in rete anche attraverso testate

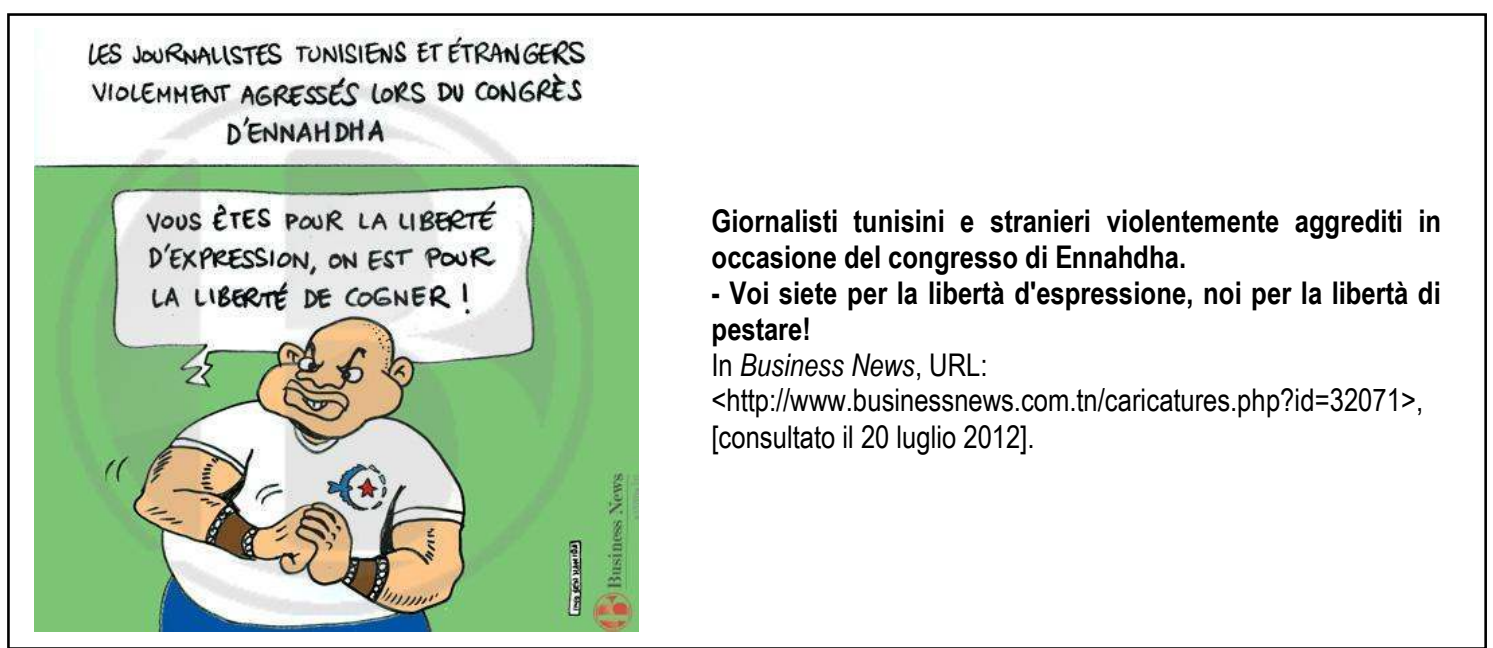

seguito dall'ex presidente del parlamento Foued Mbazaâ che gestì l'interim fino al dicembre 2011.

57 Crescenti aggressioni dei salafiti, ignorate dalle forze dell'ordine, sono ripetutamente segnalate nelle università per imporre la morale religiosa, e sono denunciate contro intellettuali e giornalisti laici. Vedi oltre, ad esempio, l'affaire Persepolis, nota 62.

${ }_{58}^{8}$ Questa e le battute che seguono sono tratte da URL:

$<$ http://zizirider.blogspot.it/p/revolution-tunisienne-humour.html > [consultato il 20 luglio 2012]. 
giornalistiche, come Businessnews 59 :

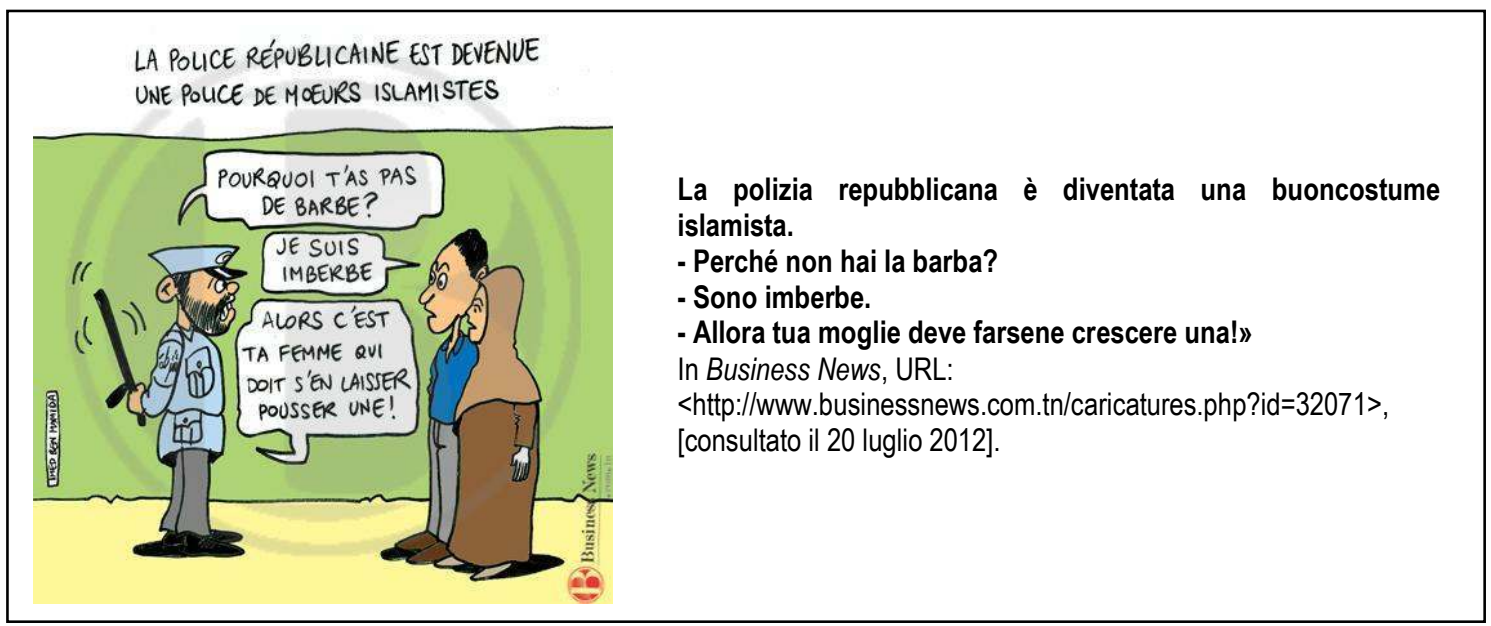

Gli islamisti sono anche il bersaglio preferito del blog di "Z" 60 :
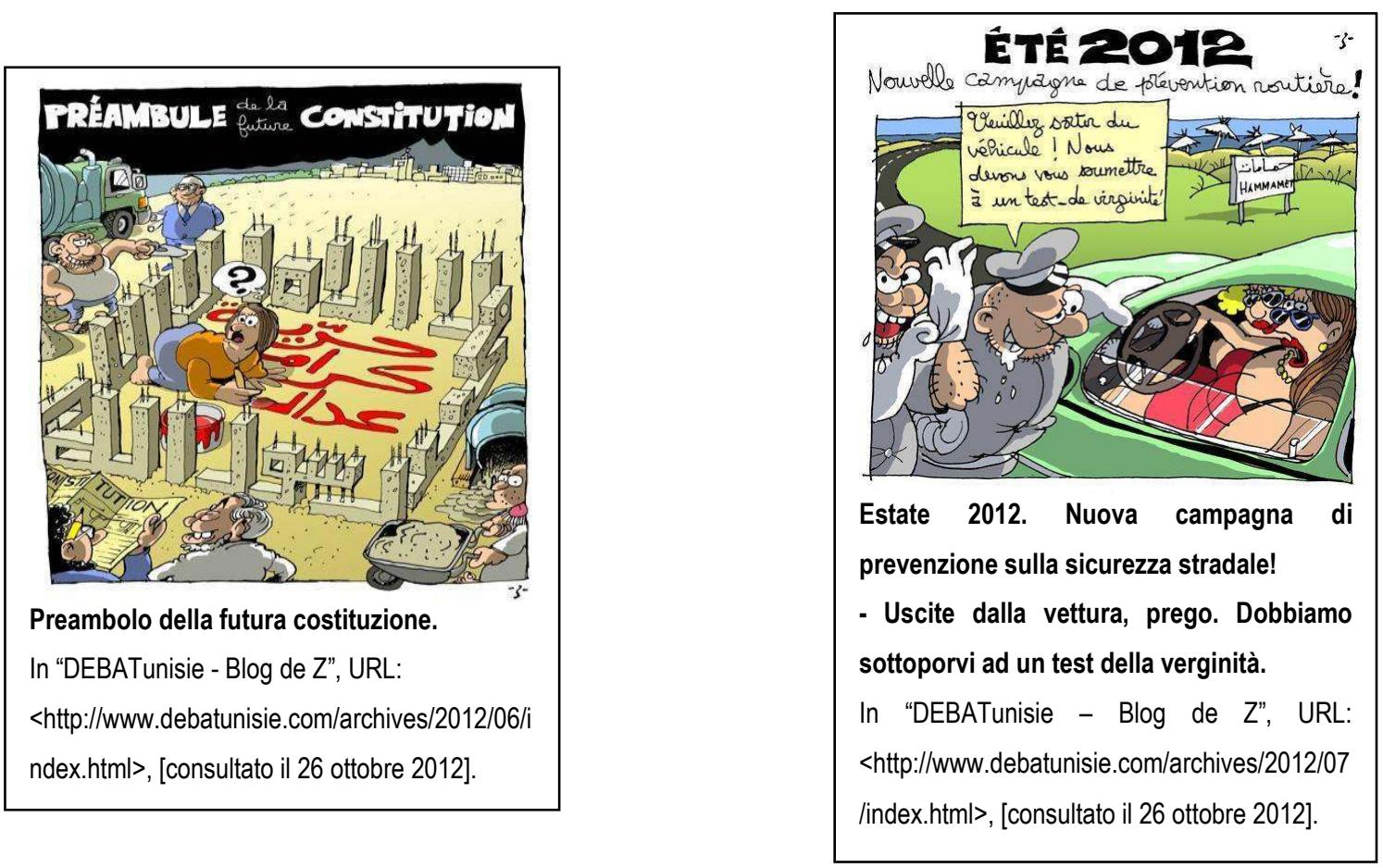

59 URL: < http://www.businessnews.com.tn/caricatures.php?id=32071 > [consultato il 20 luglio 2012].

60 'Z' é on-line con il suo blog Debatunisie (URL: < http://www.debatunisie.com/ >) circa dal 2008 e nel marzo del 2012 ha esposto le sue vignette a Ginevra per la mostra Cartooning for Peace 2012. Su "Z" vedi SLIMANI, Leïla, «Tunisie: signé -Z-, le blogueur masqué», Jeune Afrique.com, o1 février 2011,

URL : < http://www.jeuneafrique.com/Article/ARTJAJA2612po50.xmlo/ > [consultato il 29 luglio 2012]. Sul web gli si sono recentemente affiancati Bakounawar (URL: $<$ http://seifnechi.blogspot.it/>) e Willis from Tunis (URL: $<$ https://www.facebook.com/pages/WillisFromTunis/145189922203845>). Vedi anche IMEN, «(R)évolution de l'humour tunisien en ligne», 6 juin 2011.

URL: < http://www.moonsgirl.com/2011/o6/o6/revolution-de-lhumour-tunisien-en-ligne/ > [consultato il 20 luglio 2012]. 
In particolare la vignetta che fa riferimento al Preambolo della nuova Costituzione tunisina pubblicato il 4 giugno $2012^{61}$, vuole sottolineare le ambiguità del testo che, se da un lato afferma l'istituzione di uno stato democratico repubblicano, dall'altro non prefigura la laicità delle istituzioni e dichiara i principi dell'islam aperto e moderato e i valori della cultura arabo-islamica fondativi della Costituzione. L'articolo, fortemente voluto da Ennahda che ha tuttavia rinunciato ad ogni riferimento alla sciaria nel testo costituzionale, secondo alcuni commentatori potrebbe aprire lo spazio per l'introduzione del diritto religioso quale fonte giuridica, in sostituzione del forte impianto laico che ha sempre caratterizzato il paese ${ }^{62}$. "Z" raffigura una persona intenta a scrivere le parole 'libertà', 'dignità' e 'giustizia' all'interno di un recinto costituito dalla Shahada, la formula di adesione all'islam: Là ilàha illa Allah wa Muhammad rasùl Allah: «Non c’è altro dio all'infuori di Dio e Muhmmad è il suo Profeta».

A Ennahda è anche interamente dedicata una pagina Facebook, «Ennahdafacts»63, da cui sono tratte le barzellette e le vignette che seguono.

\footnotetext{
Ennahda vi augura buona notte ! Non preoccupatevi, è una delle ultime che dovrete passare con una sola moglie.
}

Ci sentiamo oltraggiati per l'immagine di Dio nel film Persepolis mandato in onda sul canale Nessma. Infatti, non ha i capelli così lunghi64.

\footnotetext{
${ }^{61}$ Il testo è tradotto in inglese e francese in: URL:

$<\quad$ http://www.jadaliyya.com/pages/index/6128/final-draft-of-preamble-to-2012-tunisianconstitut >; < http://blogs.rue89.com/tunisie-libre/2012/o6/o9/le-texte-du-preambule-de-lafuture-constitution-tunisienne-227716> . Per la versione in arabo, URL:

$<\quad$ http://www.businessnews.com.tn/Tunisie-\%E2\%80\%93-La-version-d\%C3\%A9finitive-dupr\%C3\%A9ambule-de-la-Constitution-achev\%C3\%A9e,520,31487,1 > [consultati il 25 luglio 2012].

62 Cfr., ad esempio: BELAID, Fethi, «Tunisie: le préambule de la future Constitution a été rédigé», 21 juin 2012, URL: < http://www.rfi.fr/afrique/20120608-tunisie-le-preambulefuture-constitution-ete-redige >; MEDIEN, Ahmed, «Tunisia: Final Draft of New Constitution Preamble Causes Controversy», 18 juin 2012, URL: < http://globalvoicesonline.org/2012/o6/18/tunisia-final-draft-of-new-constitution-preamblecauses-controversy/ >; BEN AMMAR, Salem, «Le préambule de la future constitution tunisienne est un acte de haute trahison», 20 juin 12, URL: < http://www.tunisienews.com/chroniques/dossier_591_preambule+future+constitution+tunisienne+est+acte+hau te+trahison.html $>$ [consultati il 27 luglio 2012].

$6_{3}<$ https://www.facebook.com/ennahdhafacts $>$ [consultati il 27 luglio 2012].

64 Nell'ottobre 2011 il canale tunisino Nessma manda in onda «Persepolis» di Marjane Satrapi, suscitando le feroci critiche da parte degli islamisti che lo considerano blasfemo e querelano il titolare del canale televisivo Nabil Karoui. In un passaggio del film di animazione, infatti, che rappresenta una dura critica al regime iraniano degli ayatollah, Dio viene raffigurato su una nuvola come un vecchio con la barba bianca, quando la religione islamica vieta la raffigurazione di immagini sacre. A testimoniare l'erosione degli acquisiti laici della Tunisia, il 3 maggio 2012 Nabil Karoui è stato condannato a una multa di $\mathbf{1 . 2 0 0}$ euro per aver «minato la morale pubblica» e per aver "diffuso informazioni che disturbano la quiete pubblica». Vedi, ad esempio: «Tunsia: Persepolis trial verdict signals 'erosion' of free speech», 3 maggio 2010. URL: $<\quad$ http://www.amnesty.org/en/news/tunisia-persepolis-trial-verdict-signals-erosion-freespeech-2012-05-03 >, [consultato 2 agosto 2012].
} 

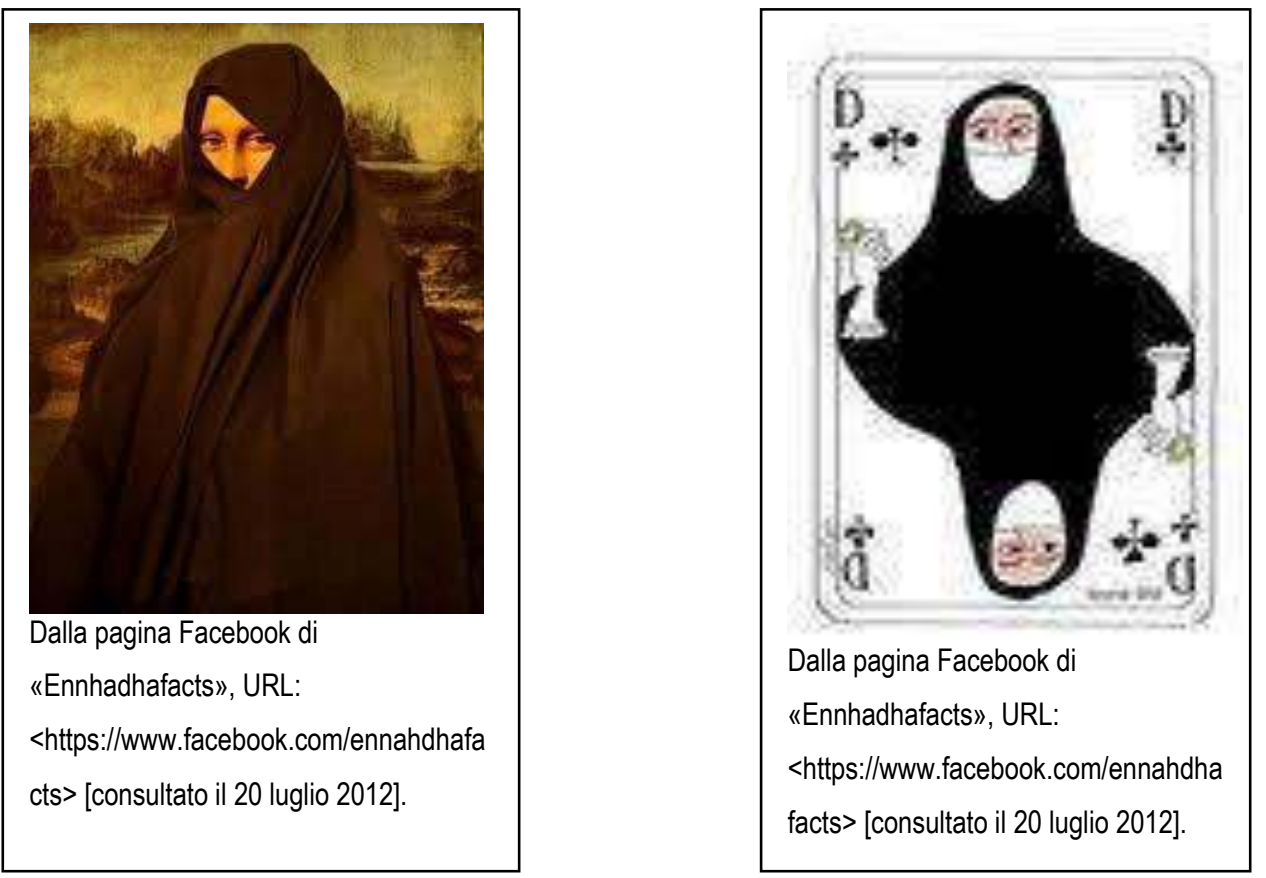

Il nesso tra satira, rivoluzione e post-rivoluzione diventa talmente stretto che a tratti, per taluni osservatori l'umorismo politico è il termometro del successo delle rivolte. Il 5 aprile 2011 il New York Times intitolava un articolo When a Punch Line is no Longer a Lifeline for Egyptians: The jokes have stalled, another sign that Egypt's revolution has too, in cui l'inviato dal Cairo Michael Slackman rilevava come in Egitto nessuno avesse più voglia di scherzare, dato il peggioramento delle condizioni di vita dopo la caduta di Mubarak, determinato dall'instabilità politica, dall'insicurezza nelle città e dalla crisi economica incrementata dal crollo del turismo ${ }^{65}$.

In realtà, come abbiamo illustrato, a distanza di oltre un anno dall'abbattimento dei regimi tunisino ed egiziano, in entrambi i Paesi la carica satirica si dimostra più vivace e creativa che mai. La ragione va probabilmente cercata in quanto scriveva Samir Shehata vent'anni prima delle rivolte, ovvero che le barzellette e in generale la satira riflettono un continuo anelito e desiderio di democrazia ${ }^{66}$, democrazia che oggi, sia in Egitto che in Tunisia, stenta ad affermarsi, tra spinte reazionarie e conservative delle vecchie guardie e rischi di involuzioni teocratiche. In tal senso, l'umorismo che le società arabe affermano quale formula di resistenza, di libertà e di rivendicazione politica sembra, mai come in questa fase della storia, riflettere l'osservazione sull'umorismo di Henri Bergson: «È qualcosa come la logica del sogno, ma d'un sogno 
che non venga abbandonato al capriccio della fantasia individuale, bensì il sogno sognato dell'intera società» 67 .

${ }^{67}$ BERGSON, Henry, Il riso. Saggio sul significato del comico, Roma-Bari, Laterza, 2009, p. 28. 


\section{* L'autore}

Barbara De Poli insegna Storia contemporanea dei paesi islamici all'Università Ca' Foscari di Venezia. Ha pubblicato, tra l'altro, I musulmani nel Terzo Millennio (Roma, Carocci, 2007) e Il sorriso della mezzaluna (Roma, Carocci, 2011).

URL: < http://studistorici.com/progett/autori/\#DePoli >

\section{Per citare questo articolo:}

De POLI, Barbara, «Dal dissenso alla rivoluzione: satira e potere nel mondo arabo contemporaneo», Diacronie. Studi di Storia Contemporanea: La satira fa storia. Eventi, pratiche, linguaggi, 29/10/2012,

URL:<http://www.studistorici.com/2012/10/29/depoli_numero_11/ >

Diacronie Studi di Storia Contemporanea $\beta$ www.diacronie.it

Risorsa digitale indipendente a carattere storiografico. Uscita trimestrale. redazione.diacronie@hotmail.it

Comitato di redazione: Marco Abram - Giampaolo Amodei - Jacopo Bassi - Luca Bufarale - Gianluca Canè - Alessandro Cattunar - Alice De Rensis - Barbara Galimberti - Deborah Paci - Fausto Pietrancosta - Matteo Tomasoni - Luca Zuccolo

Diritti: gli articoli di Diacronie. Studi di Storia Contemporanea sono pubblicati sotto licenza Creative Commons 2.5.

Possono essere riprodotti a patto di non modificarne i contenuti e di non usarli per fini commerciali. La citazione di estratti è comunque sempre autorizzata, nei limiti previsti dalla legge. 